\title{
Tidal forces and variability in close binary stars: the $\boldsymbol{\beta}$-factor
}

\author{
Gloria Koenigsberger, Edmundo Moreno, and Fausto Cervantes \\ Centro de Ciencias Físicas, Instituto de Astronomía, UNAM, \\ Apartado Postal 70-264, Ciudad Universitaria, 04510 México D.F., \\ México
}

\begin{abstract}
We present the results of calculations that illustrate the effects, on orbital timescales, produced on the external layer of a star that is in a binary system in which the stellar rotation period is not synchronized with the orbital period. The calculations show how, for a fixed set of parameters, the amplitudes of the oscillations depend on stellar radius and on $\beta$, the ratio between the stellar rotation angular velocity and the orbital angular velocity.
\end{abstract}

\section{Introduction}

In a close binary system, the gravitational field of each of the stars acts as an outer perturbing force on its companion, and through the resulting tidal interaction the stellar surfaces are deformed with respect to their unperturbed shape. If the system is in equilibrium, this shape remains constant throughout the orbital cycle. However, if the stellar rotation period is not synchronized with the orbital period, the deformation varies as a function of time (see, for example, Press \& Teukolski 1977; Zahn 1977; Savonije, Papaloizou \& Alberts 1995; Kumar, Ao \& Quataert 1995; Goldreich \& Nicholson 1989). In circular orbits in which $P_{\text {orb }} \neq P_{\text {rot }}$ and in eccentric orbits, in general, this variability will occur.

Motivated by the peculiar eruptive behavior of the Wolf-Rayet/LBV system HD 5980 (see Koenigsbergeret al. 2000 and references therein), we have developed a simple model (Moreno \& Koenigsberger 1999) which provides insight into some of the effects that are produced by tidal forces on the surface of massive stars and that could lead to unexpected and brief episodes of mass ejection. We are interested in variability on orbital timescales. The model calculates the solution of the equations of motion for one layer of surface elements distributed along the equator of the star, as they respond to the forces in the system. These forces include the gravitational forces of both stars, gas pressure, centrifugal and coriolis forces. Radiation pressure forces are neglected. The model permits calculations for arbitrary rotational velocities and includes the effects of viscosity. The stellar rotation is characterized by the parameter $\beta=\omega / \Omega$, where $\omega$ and $\Omega$ are the stellar rotation angular velocity and the orbital angular velocity, respectively. The equators of both stars are assumed to lie in the orbital plane. The computation consists of solving the equation of motion for a given number (usually between 200-1000) of surface elements located along the equator in a 

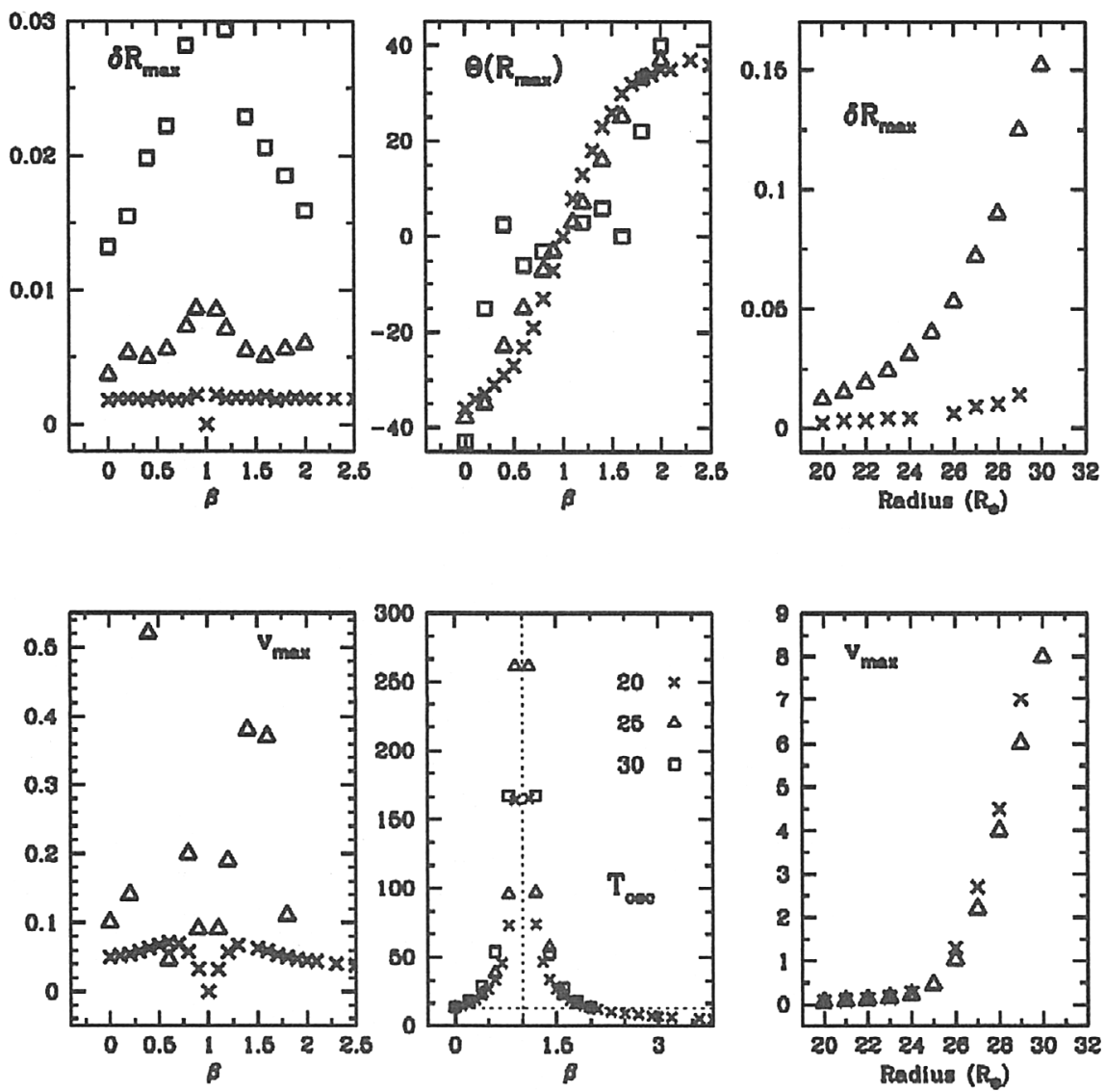

Figure 1. The dependence of oscillations quantities in an $e=0$ binary system as a function on $\beta$-for three fixed values of the stellar radius (left and middle panels) and the dependence of stellar radius for two fixed values of $\beta$ (right panels). The orbital period of this system is $13 \mathrm{~d}, M_{\mathrm{A}}=M_{\mathrm{B}}=40 \mathrm{M}_{\odot}$. Velocities are in $\mathrm{km} \mathrm{s}^{-1}$, periods in days, and displacements in $R_{\odot}$.

thin shell. This thin shell lies deep enough within the atmosphere so that the oscillations can be treated as adiabatic, but high enough so that the amount of mass lying above it is negligible. The main body of the star, interior to the shell, is assumed to behave as a rigid body and the tidal deformation is assumed to occur mainly in the external shell.

\section{Results}

The overall behavior of the stellar surface can be characterized by the displacement of each surface element from the equilibrium position it would assume in the absence of the external perturbing force. The results for each model calculation are described by the following quantities:

(i) $R_{\max }$, the maximum radius achieved by the star at a given surface element 

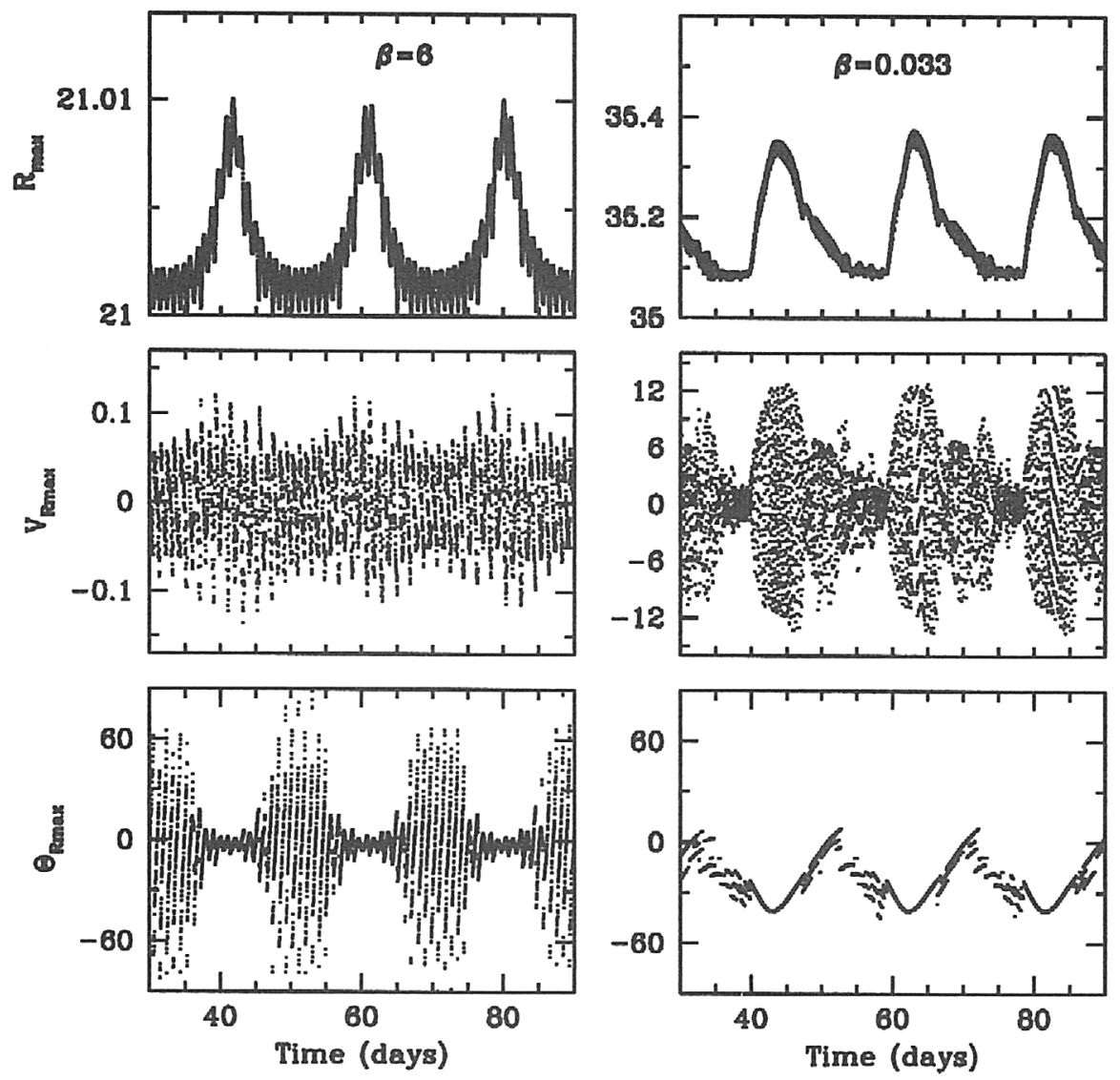

Figure 2. Oscillations of a $50 \mathrm{M}_{\odot}$ star, whose companion is $28 \mathrm{M}_{\odot}$, and in which the orbit is eccentric $(e=0.27$, and has a period 19.3d. Left panel: $R_{\mathrm{A}}=21 \mathrm{R}_{\odot}$ and $\beta=6$ at periastron; Right panel: $R_{\mathrm{A}}=35 \mathrm{R}_{\odot}$ and $\beta=0.033$.

at any given time. Depending on the stellar angular rotation rate, within an orbital cycle, this maximum displacement can occur at any of the surface elements. The tidal bulge coincides with the surface elements associated with and including the element at which $R_{\max }$ occurs;

(ii) $\Theta_{R_{\max }}$, the angle between the line joining the centers of the two stars and the radius vector of the surface element at which $R_{\max }$ occurs. The tidal bulge does not necessarily point in the direction of the companion;

(iii) $\delta R_{\max }$, the amplitude of oscillation in the values of $R_{\max }$;

(iv) the maximum radial velocity, $V_{\max }$, achieved at any one of the surface elements;

$(v)$ the radial velocity, $V_{R_{\max }}$, of the element at which $R_{\max }$ occurs;

(vi) $T_{\text {osc }}$, the characteristic timescale for the oscillations of $R_{\max }$. Note that since $R_{\max }$ may occur at any of the surface elements used in the calculation, the oscillations in $R_{\max }$ do not necessarily correspond to the oscillations of any one given surface element. 
For circular orbits, when $\beta=1$ (i.e., corotation), we recover the classical result of the equilibrium tide, with the tidal bulge pointing in the direction of the companion (i.e., $\Theta_{R_{\max }}=0$ ). For values of $\beta<1$ and $\beta>1, R_{\max }$ and $V_{\max }$ present oscillations with amplitudes and periods that depend on the value of $\beta$ and the value of the stellar radius, for a given set of stellar and orbital parameters. The oscillations display double-wave patterns with periods that are longer than the orbital period, tending asymptotically to $P_{\text {orb }}$ as $\beta$ approaches zero or becomes very large. The amplitudes of the oscillations depend strongly on $\beta$ and on the stellar radius. The largest displacements are found for values of $\beta$ close to unity (excluding, however, $\beta=1$, where no oscillations are present), and the amplitudes grow rapidly with increasing stellar radii. The velocities also depend strongly on the stellar radius. In Figure 1 we illustrate these trends for the case in which two stars of equal masses $\left(40 \mathrm{M}_{\odot}\right)$ are in a circular orbit of radius $100 R_{\odot}$. The left and middle panels show the result of varying the value of $\beta$ while holding the radius of the star fixed, for three different values of $\mathrm{R}$ (crosses: $20 \mathrm{R}_{\odot}$; triangles: $25 \mathrm{R}_{\odot}$; squares: $30 \mathrm{R}_{\odot}$ ). The right panels illustrate the variation of displacements and velocities, as a function of stellar radius, for two fixed values of $\beta$ (crosses: 0.5 ; triangles: 1.5). For large values of $\beta$ and/or the stellar radius, the contact between adjacent surface elements may be lost, thus ending the computation. For example, at $R_{*}=25 \mathrm{R}_{\odot}$, we were unable to continue the calculation for $\beta>2$.

For eccentric orbits, it is important to note that even if the system is corotating at periastron, $\beta$ differs from unity at all other orbital phases. The varying orbital separation, as a function of phase, introduces an additional factor in the analysis. Figure 2 illustrates the results of a calculation for a binary system having masses $M_{\mathrm{A}}=50 \mathrm{M}_{\odot}, M_{\mathrm{B}}=28 \mathrm{M}_{\odot}, e=0.27$, and a semi-major axis for the orbit of $100 \mathrm{R}_{\odot}$ for two different values of $R_{A}$. The maxima which are observed in the top panels of Figure 2 coincide with the periastron passages, but note the oscillations that are present, with timescales of approximately 1 day on the left panels $\left(\beta=6 ; R_{\mathrm{A}}=21 \mathrm{R}_{\odot}\right)$. For this case, the radial velocity of the $R_{\max }$ elements is $\sim 0.1 \mathrm{~km} \mathrm{~s}^{-1}$. Near periastron passage, the tidal bulge points very nearly in the direction of the companion, although at other orbital phases it can point in any other direction. In the right panels, $R_{\mathrm{A}}=35 \mathrm{R}_{\odot}$, but in this case it was not possible to carry out the computation for values of $\beta$ greater than 0.033 , since the surface elements immediately lose contact for larger values. The behavior of $R_{\max }$ can be described as an abrupt rise to maximum, which is achieved slightly after periastron passage, with a gradual decline towards apastron. The radial velocities in this case are orders of magnitude larger than in the case of a smaller radius, with values as high as $12 \mathrm{~km} \mathrm{~s}^{-1}$. The location of the tidal bulge varies between $-45^{\circ}$ and $0^{\circ}$; i.e., it is 'lagging behind' during most of the orbital cycle. This illustrates the strong dependence of the oscillations on stellar radius.

If the erupting star in the HD 5980 binary system is a rapid rotator (Koenigsberger et al. 2002: $v \sin i \simeq 200 \mathrm{~km} \mathrm{~s}^{-1}$ ) that has just left the Main Sequence, then it is very likely that tidally-induced oscillations, with growing amplitudes due to the increasing radius of the star, may have played a significant role in driving the large mass outflow observed during the 1994 eruption. It is also possible that these effects are responsible for currently observed shorter timescale variability (Villar-Sbaffi et al. 2003). 


\section{Conclusions}

Our simple model predicts that in close binary systems that are not in an equilibrium configuration, the stellar surface is far from being described in terms of a stationary structure, as show to be the case by other theoretical approaches. Assuming that the amplitudes of oscillation may be associated with activity on the surface of the star, the model predicts that there should be a trend in the indicators of stellar surface activity, such as X-ray emission, that follows the trends illustrated in Figure 1 for the dependence on $\beta$. That is, stronger X-ray emission might be expected in systems where $\beta \neq 1$, compared to similar systems with $\beta=1$. Cyclical behavior, with periods different from the orbital period are also predicted. In addition, a star undergoing a rapid expansion becomes unstable and undergoes an episode of significant mass-loss long before it reaches the dimensions of the Roche Lobe, simply due to the oscillations induced by the tidal forces. Thus, the assumption of Roche Lobe Overflow in close binary systems may not be a valid one in general.

\section{References}

Goldreich, P., Nicholson, P.D. 1989, ApJ 342, 1079

Koenigsberger, G., Georgiev, L., Barbá, R., Tzvetanov, Z., Walborn, N.R., Niemela, V., Morrell, N., Schulte-Ladbeck, R.E. 2000, ApJ 542, 428

Koenigsberger, G., Kurucz, R., Georgiev, L. 2002, ApJ 581, 598

Kumar, P., Ao, C.O., Quataert, E.J. 1995, ApJ 449, 294

Moreno, E., Koenigsberger, G. 1999, RMxAA 35, 157.

Press, W.H., Teukolsky, S.A. 1977, ApJ 213, 183

Savonije, G.J., Papaloizou, J.C.B., Alberts, F. 1995, MNRAS 277, 471

Villar-Sbaffi, A., Moffat, A.F.J., St-Louis, N. 2003, these Proceedings

Zahn, J.-P. 1977, A\&A 57, 383

\section{Discussion}

LANGER: Are your results affected by whether or not the forced oscillation frequency corresponds to an eigen-frequency of the oscillating star?

KOENIGSBERGER: If a resonance frequency is encountered, the amplitudes of the oscillations increase abruptly, consistent with what is expected from theory and other treatments of the problem.

MAEDER: This $\beta$-factor is a nice (new) parameter. If we consider a significant sample of massive binaries, do we have an idea of the distribution of the $\beta$-values?

KOENIGSBERGER: I don't have a significant sample of massive binaries as yet, but from the data I have so far the range in $\beta$ is $\sim 0.4-3$. It is crucial for more values of $v \sin i$ to be obtained, particularly for those systems where significant variability is present. 